\title{
Thermal conductivity of food products using: A correlation analysis based on artificial neural networks (ANNs)
}

\author{
Ajasa, Abiodun Afis* , Adenowo, Adetokunbo Abayomi, Ogunlewe, Adeyinka Oluremi, \\ Folorunso, Comfort Oluseyi
}

Department of Electronic and Computer Engineering, Faculty of Engineering, Lagos State University, Epe, Lagos, Nigeria

\section{Email address:}

ajasaaf@yahoo.com (Ajasa A. A.), adetokyom@yahoo.com (Adenowo A. A.), yogunlewe@yahoo.com (Ogunlewe A. O.), comfortobolajoko@yahoo.com (Folorunso C. O.)

\section{To cite this article:}

Ajasa, Abiodun Afis, Adenowo, Adetokunbo Abayomi, Ogunlewe, Adeyinka Oluremi, Folorunso, Comfort Oluseyi. Thermal Conductivity of Food Products using: A Correlation Analysis Based on Artificial Neural Networks (ANNs). Advances in Bioscience and Bioengineering. Vol. 2, No. 2, 2014, pp. 14-24. doi: 10.11648/j.abb.20140202.12

\begin{abstract}
This paper presents the correlation between the predicted and desired/targeted thermal conductivity of food products as a function of moisture content, temperature and apparent density. The food products considered in this work are the bakery products which include bread, bread dough, cake, and whole-wheat dough. Statistical data of results from previous work in existing literatures were used in this work for a wide range of moisture contents, temperatures and apparent densities resulting from baking conditions. The results of this work showed straight line curves when the predicted values of thermal conductivity were plotted against the targeted values of thermal conductivity. This demonstrates correlation between the predicted and targeted thermal conductivities when the points are joined together (best fit-points), hence, a very good agreement between the predicted and the desired values of thermal conductivity. The two ANN models that were finally selected, after several configurations had been considered and evaluated, are the optimal ANN model that was found to be a network with two hidden layers and eight neurons and the simplest ANN model was equally found to be a network with one hidden layer and ten neurons. The estimated errors between the predicted and desired (or targeted) thermal conductivity values of the bakery products for both the optimal ANN and simplest ANN models are the MRE, MAE and SE. Moreover, the results also showed that the optimal ANN model had an MRE of $0.04878 \%$, an MAE of $0.0054 \mathrm{~W} / \mathrm{mK}$ and an SE of $0.0015 \mathrm{~W} / \mathrm{mK}$ while the simplest ANN model was estimated to have an MRE of $0.03388 \%$, an MAE of $0.0034 \mathrm{~W} / \mathrm{mK}$ and an SE of $0.0011 \mathrm{~W} / \mathrm{mK}$. These errors are approximately equal to zero (i.e., $0 \mathrm{~W} / \mathrm{mK}$ ) and could, therefore, be regarded as a good result for the prediction. Since the simplest ANN model had the least values of all three errors (MRE, MAE and SE) when compared with other configurations, including the optimal ANN model, it is, however, regarded as the best ANN model and is, thus, recommended.
\end{abstract}

Keywords: Thermo-Physical Properties of Biological Products, Thermal Conductivity of Bakery Products, Back-Propagation, Artificial Neural Network, Mean Absolute Error, Mean Relative Error, Standard Error

\section{Introduction}

In a typical baking process, bakery products undergo physical, chemical and biochemical changes that cumulatively result in expansion of bulk volume, evaporation of water, formation of a porous structure, denaturation of protein, gelatinization of starch, formation of crust and browning reactions respectively. During such processes, ovens powered by gas, electricity, firewood, charcoal, or microwaves are used for generating the required heat.

The thermal conductivity of a material can be defined as the ability of a substance to conduct heat. It is a property of materials that expresses the heat flux, $\mathrm{F}\left(\mathrm{W} / \mathrm{m}^{2}\right)$ that will flow through the material if a certain temperature gradient $\Delta \mathrm{T}(\mathrm{K} / \mathrm{m})$ exists over the material. Thermal conductivity of materials can be defined as the heat flow per unit area per unit time when the temperature decreases by one degree in unit distance. 
Accordingly, heat is transferred mainly by convection from the heating media, and by radiation from oven walls to the product surface and then by conduction to the geometric centre. At the same time, moisture diffuses outward to the product surface $[1,5]$.

The problem of interest in the design of bakery ovens is concerned with the promotion of the required rate of heat transfer with the minimum possible surface area and temperature difference. And from the engineer's point of view, it is usually sufficient to know the total quantities of energy emitted and absorbed by the material at various temperatures [16]. As such, it is frequently necessary to establish the rate at which heat will be conducted through a solid if a known temperature difference exists across the solid. For such purposes, and especially if the process varies with time, sophisticated mathematical techniques are required to establish this, the phenomenon being known as transient-heat conduction. A knowledge of the product properties, including thermal conductivity as a function of processing conditions is needed in order to predict the temperature and water distribution in the product during baking $[5,8]$. The temperature and moisture distribution within the porous product can be predicted using diffusion equations of heat and water.

\section{Literature Review}

Neural networks have been trained to perform complex functions in various fields including pattern recognition, identification, classification, speech, vision, and control systems. Today, neural networks can be trained to solve problems that are difficult for conventional computers or human beings. Commonly, neural networks are adjusted (or trained) so that a particular input leads to a specific target output. Typically, many such input/target pairs are needed to train a neural network.

Linko and Zhu also stated that of all the various modelling approaches of predicting the thermal conductivity of a wide range of foods, including bakery products, the neural network-based models have proven to be excellent. Amongst the major benefits of using ANN are excellent management of uncertainties, noisy data and nonlinear relationships. Neural network modelling has generated increasing acceptance and is an interesting method in the estimation, prediction and control of bioprocesses [9].

Review of past work showed that Ruan et al. applied ANN modelling to predict the rheological properties of dough in 1995 [15]. Fang et al. also applied the ANN modelling to predict the physical properties of ground wheat in 1998 [18] while Hussain and Rahman, in 1999, predicted the thermal conductivity of fruits and vegetables with the application of ANN modelling [6]. Similarly, ANN modelling was applied by Myhara et al. in 1998 for the prediction of isotherms of dates [11], Ni and Gunasekaran in 1998 [7] and Xie and Xiong in 1999 [13] also applied
ANN modelling differently for the prediction of food quality. Recently, Sablani and Shayya in 2001 applied ANN modelling for the prediction of heat penetration parameters in Stumbo's method of thermal process calculations [20].

Rahman's model [4] (data considered only above $0^{\circ} \mathrm{C}$ ) predicted thermal conductivity with mean relative errors of 24.3 and $81.6 \%$, respectively. This model was able to predict thermal conductivity with a mean relative error of $12.6 \%$ and a mean absolute error of $0.081 \mathrm{~W} / \mathrm{mK}$. The model can be incorporated in heat transfer calculations during food processing where moisture, temperature and apparent porosity dependent thermal conductivity values are required.

Shyam et al. (2002) optimal ANN model was found to be a network with 6 neurons in each of the 2-hidden layers. This optimal model was capable of predicting the thermal conductivity values of various bakery products (such as bread, bread dough, French bread, yellow cake, tortilla chip, whole wheat dough, baked chapatti and cup cake) for a wide range of conditions with a mean relative error of $10 \%$, a mean absolute error of less than $0.02 \mathrm{~W} / \mathrm{m} \mathrm{K}$ and a standard error of about $0.003 \mathrm{~W} / \mathrm{m} \mathrm{K}$. The simplest ANN model, which had 1-hidden layer and 2 neurons, predicted thermal conductivity values with a mean relative error of less than $15 \%$ [3]. All these work were successfully carried out with satisfactory results obtained using ANN modelling.

In predicting thermal properties of a material at desired conditions, several modelling approaches have been proposed and none of them was found suitable for use over a wide range of foods. According to Murakami and Okos (1989) the most promising approach is based on chemical composition, temperature and physical characteristics [12]. More recently, Baik et al. in 2001 reviewed common and new measurement techniques, prediction models and published data on thermo-physical properties of bakery products [2].

The series model of specific heat, density and thermal diffusivity has been successfully applied to many food materials including porous materials such as baking products. However, for the prediction of thermal conductivity of porous food, there is still some theoretical argument for the use of the structural models [12]. Murakami and Okos (1989) evaluated nine different structural models with specific types of porous foods and found that parallel and perpendicular models showed 12-97\% and $18-61 \%$ standard errors respectively.

Among the models, Keey's model was found to be the best prediction model for porous grains and powders. The model produced standard errors of $<28 \%$ for full fat dry milk and $<10 \%$ for other food materials. In addition, all structural models neglect interactions between components, phase transition and distillation heat transfer, which may be significant in the baking process [1]. Hence, most thermal conductivity models reported are usually empirical rather than theoretical. 


\section{Methodology}

In this section, the various materials and methods used are discussed here.

\subsection{Materials}

Basically, baked products made by bakeries can be divided into three (3) different groups:

- $\quad$ Bread loaves (e.g. Wheat bread, rye bread, specialty bread)

- $\quad$ Small baked products (e.g. rolls, buns, croissants)

- $\quad$ Fine baked goods (e.g. plaits, apple strudel, biscuits, salts pretzels)

Depending on the specific type of bakery product to be produced and associated production process, a wide variety of raw materials are available to the baker. In addition to the basic Raw materials (flour, water, salt and yeast) various other ingredients can be used. The ingredients used have an $\mathrm{n}$ influence on the characteristics or technological aspect of the dough.

Flour

Flour account for the main portion of the raw materials involved in baked product production and the thermal conductivity of flour need to be considered while applying the neural network. Flour used is mainly those extracted from two basic cereals - wheat and rye. Flour from other grains which do not contain gluten-forming proteins is usually blended with wheat flour for the production of bakery products. The quality of flour is basically dependent on its intended use. The flour quality depends on the following factors:

- Wheat variety

- Growing conditions

- Grain storage

- Flour production technique

- Flour storage

Table 1. Example of a requirement profile for various baking flours

\begin{tabular}{lllll}
\hline & Patent & gluten \% & High-Ash & whole-Grain \\
\cline { 2 - 5 } & Flour & Flour & Flour & Flour \\
\hline Moisture \% & $13.0-15.0$ & $13.0-15.0$ & $13.0-15.0$ & $11.0-13.0$ \\
Ash \% DM & $0.38-0.60$ & $0.64-0.78$ & $1.05-1.15$ & $1.75-1.95$ \\
Protein \% & $12.0-14.0$ & $13.5-15.0$ & $14.0-15.5$ & $13.5-15.0$ \\
Wet gluten \% & $28.0-33.0$ & $31.0-35.0$ & $32.0-36.0$ & $29.0-33.0$ \\
Falling N umber sec & $320-410$ & $300-390$ & $280-380$ & $300-380$ \\
Sedimentation ml & $38-45$ & $38-43$ & $25-30$ & NIL \\
Water Absorption \% & $60-64$ & $61-65$ & $65-70$ & $66-71$ \\
Weakening FU & $20-70$ & $60-90$ & $60-90$ & $60-90$ \\
Dough energy cm2 & $90-130$ & $80-110$ & $55-85$ & $60-90$ \\
Max. viscosity AU & $500-1000$ & $350-800$ & $300-550$ & $250-500$ \\
\hline
\end{tabular}

Average patent flour (first grade) is made up of the following:

- Carbohydrates: $73.5 \%$. This includes starch: $71 \%$, soluble sugars $2.4 \%$ and cellulose $0.1 \%$.

- Proteins: $11.0 \%$ :This includes gluten-forming proteins $10 \%$ \& water soluble proteins $1 \%$

- Water: $14 \%$

- Fat: $1.0 \%$

- Minerals: $0.5 \%$

In terms of quantity, carbohydrates account for the greatest portion in flour which incidentally forms the greater part of bakery products. Starch essentially fulfills the following functions: (1) a source of nutrient for yeast after enzymatic degradation, (2) absorption of free dough water during gelatinization, (3) contribution to crust, crumb and coloration formation.

The content of soluble sugar substances in wheat flour amounts to approximately $1.5-3 \%$. The main soluble sugar substances are glucose, maltose and dextrin. They are dissolved during dough production in the available dough liquid. Glucose and Maltose are available as yeast food while Dextrin cannot be fermented by yeasts
Thermal properties (thermal conductivity and diffusivity) of gluten and glutenin were measured in the temperature range $60-175^{\circ}$ typically used in extrusion processing. Thermal conductivity and diffusivity of gluten decreased with increasing temperature and increased with increasing moisture content. Thermal conductivity and diffusivity of glutenin increased with temperature and moisture content. Thermal conductivity of gluten was $0.06-0.35 \mathrm{~W} / \mathrm{m}-\mathrm{C}$ and glutenin was $0.29-0.49 \mathrm{~W} / \mathrm{m}-\mathrm{C}$ for the temperature range $60-175^{\circ}$ and moisture content range of $0-30 \%$.

\subsection{Measurement of Thermal Conductivity}

Thermal conductivity of materials can be measured and obtained through different materials. Depending on the measurement time these methods can be classified as either;

- Steady State or

- Transient or Unsteady

These two methods are explained below:

A. Steady State Method

In the steady state methods, the measurement time can be much longer for poor thermally conductive samples than in the transient methods. During testing, moisture migration 
and property changes can occur due to long exposure to high temperatures. Thus, this method may not be suitable for measurement of the thermal conductivity of bakery products. The steady state methods include:

- Guarded hot plate

- Differential scanning calorimeter (DSC) attachment method

- Capped column test device

(i) Guarded hot plate Method

This method is a representative steady state method. A time- independent heat flow is generated through the sample between a heat source and a heat sink. This method is mathematically simple to process, and it is easy to control experimental conditions. The $\mathrm{k}$ values can be calculated as a mean value measures over the temperature interval used in the experiment using Fourier's heat conduction equation. Experimental uncertainty in the measured thermal conductivity of biscuit dough was $\leq 7.4 \%$ using a similar method, single-plate method [22]. This evaluation was based on combination of instrumentation errors, geometrical uncertainties, and deviations from the assumed one-dimensional nature of heat flow.

(ii) DSC Attachment Method

In order to alleviate the disadvantage of conventional steady state method, an attachment to a DSC was constructed by Buhri and Singh [23]. The main innovations are relatively rapid measurement (10 to $15 \mathrm{~min}$ ), small sample size, and no risk of drying of the samples. The thermocouple probe was inserted into the sample, and the DSC heating pan temperature was kept at $40^{\circ} \mathrm{C}$. After 5 minutes the sample initial temperature was recorded. The pan temperature was then immediately increased by $10^{\circ} \mathrm{C}$. After 10 to 15 minutes, a new steady state existed, and the final sample temperature was recorded. The $\mathrm{k}$ was obtained using the following equation, which is based on Fourier's heat conduction equation:

$$
k=\frac{L \Delta Q}{A\left(\Delta t_{2}-\Delta t_{1}\right)}
$$

where $\mathrm{k}=$ thermal conductivity, $\mathrm{L}=$ sample length,

$\Delta \mathrm{Q}=$ difference of energy required to maintain pan temperature,

$\mathrm{A}=$ sample area perpendicular to heat flow,

$\Delta \mathrm{t}_{2}=$ final temperature difference between DSC heating pan and sample

$\Delta \mathrm{t}_{1}=$ Initial temperature difference between DSC heating pan and sample

(iii) Capped column test device

The use of a capped column test device was introduced by Zhou et al [24]. Experiments with the capped column test device were conducted with constant heat flux and no net water loss. Constant heat flux was provided by circulating hot cold water at constant temperatures at the two ends of the cylindrical test sample (diameter $3 \mathrm{~cm}$, height $5 \mathrm{~cm}$, and $2.5 \mathrm{~cm}$ ). The test sample was enclosed in polystyrene foam to minimize heat loss to the surroundings. To eliminate the gravity-induced moisture migration effect, the capped column test device was operated horizontally.

Temperature distribution along the height of the test sample water stream was measured. The moisture content distribution of test sample was determined by measuring moisture content of the sample cut into several section of equal height. The $\mathrm{k}$ value can be determined by applying a heat and mass transfer equation to the experimental temperature and moisture gradient data. The measurement is fast (within several minutes), and there is no worry about experimental deviation because of the moisture gradient, because the measurement device and data analysis are designed to evaluate thermal and mass transfer properties simultaneously.

B. Transient Method

The transient techniques are most suitable for bakery products because testing is very fast and it yields more accurate results. In the transient method, the sample is subjected to a time-dependent heat flow and the temperature is measured at one or more points within the sample or at its surface. The transient technique includes:

- $\quad$ Line heat source probe method

- Temperature history

- Transient hot strip method (THS)

(i) Line Heat Source Probe Method

This method is recommended for most food applications, including bakery products. The method is simple and fast (measuring times are between 3 and 600s) and requires relatively small samples, but it does require a fairly sophisticated data acquisition system. The probe $(0.66 \mathrm{~mm})$ consists of a constantan heater wire and chromelconstantan thermocouple wire.

A line heat source probe is embedded in the sample (regarded as an infinite body), which is initially at a uniform temperature, resulting in a cylindrical temperature distribution. Heating and temperature monitoring occur simultaneously. The rate of temperature rise of the heater is directly related to the sample's conductivity. The slope of the linear portion of each data set was used to determine effective thermal conductivity by equation (2)

$$
k=Q \frac{\operatorname{In}\left(\frac{\tau_{2}}{\tau_{1}}\right)}{4 \pi\left(t_{2}-t_{1}\right)}
$$

where $\mathrm{k}=$ thermal conductivity of the sample,

$\tau_{1}=$ initial time when probe heater was energized,

$\tau_{2}=$ final time since probe heater was energized,

$t_{1}=$ temperature of probe thermocouple at $t_{1}$,

$t_{2}=$ temperature of probe thermocouple at $t_{2}$,

$\mathrm{Q}=$ heat flux generated by probe heater.

In order to obtain correct results and avoid edge effects in a line heat source probe method, the following constraints [25] should be considered:

$$
\text { Probe size: } r_{p}<2 / 5 \sqrt{ }\left(\frac{\alpha \tau}{2}\right)
$$

where $r_{p}=$ probe radius, $\alpha=$ thermal diffusivity of the sample, $\tau=$ time, test duration 


$$
\text { Size of sample: } r_{s}>2.6 \sqrt{\alpha \tau}
$$

where $r_{s}=$ sample radius

Other experimental errors can be produced by inserting a probe into unstable structure because this may rupture the structure close to the probe, giving rise to false values. Thus, the use of a linear movement probe header is strongly recommended for thermal conductivity measurement using this method.

\section{(ii) Temperature History}

This method is suitable for thermal conductivity of porous, fine particles grains or beans. By measuring the temperature profile in a product subject to a known energy flow, $\mathrm{k}$ and $\alpha$ can be calculated ( $\mathrm{k}=$ thermal conductivity and $\alpha=$ thermal diffusivity). This method requires rather long measuring times, from 10 to 200 minutes. For bakery products, this method could be applied to bread dough and tortilla.

(iii) Transient Hot Strip Method (THS)

A thin foil of platinum or nickel serves both a heat source and a temperature sensor. A constant current is supplied to the metal foil and the voltage increase is monitored over a short period; typically 5 seconds. The voltage change is due to the temperature increase, normally less than $1{ }^{\circ} \mathrm{C}$, which causes an increase in the electrical resistance of the metal strip. The thermal properties of the surrounding material determine the temperature change in the material foil. The THS method has been used mostly for building materials and liquids [26], but it was also applied measuring the thermal conductivity of potatoes, meat and dough [27]. The time needed for one measurement often has to equilibrate before the test can start.

\subsection{Methods}

(i) Mathematical Modelling for Thermal Conductivity of Bakery Products

Although the exact mechanism of heat conduction in solids is not entirely understood, it is believed, however, to be partially due to the motion of free electrons in the solid matter, which transport energy if a temperature difference is applied (Refer to Fig.1) and the conceptual representation of Oven dynamics during typical baking process as depicted in figure 2 below.

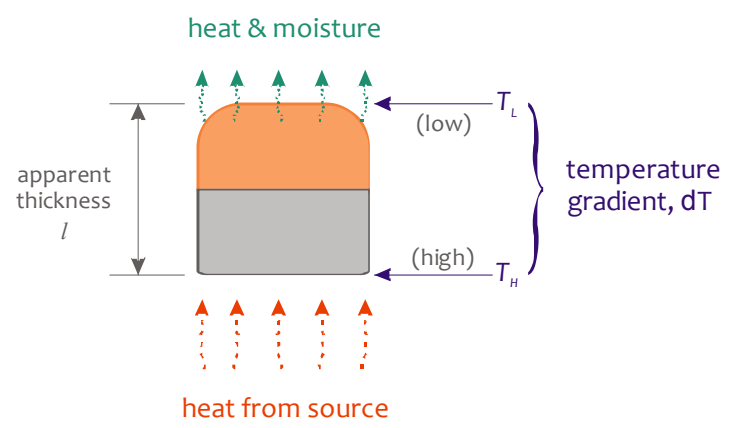

Fig. 1. Conceptualisation of thermal conductivity of a bakery product

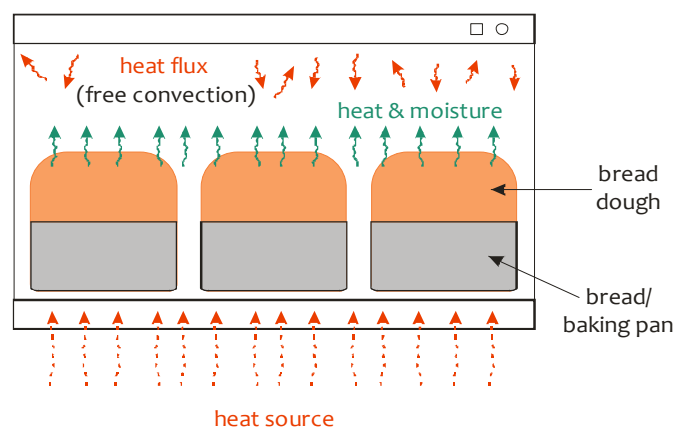

Fig. 2. Conceptual representation of Oven dynamics during typical Baking process

From Fourier's law of heat conduction, the rate at which such heat is conducted through a body per unit crosssectional area is said to be proportional to the negative of the temperature gradient existing in the body [16]. In other words,

$$
\mathrm{Q} \propto-\nabla \mathrm{T}
$$

The proportionality factor is called the thermal conductivity of the material. By definition, it is the ability of the material to conduct heat and thus, a measure of the rate at which heat flows through a material between points at different temperatures, measured in watts per meter per degree.

As a property, the thermal conductivity expresses the heat flux, $Q\left(\mathrm{~W} / \mathrm{m}^{2}\right)$ that will flow through the material if a certain temperature gradient, $\Delta T(\mathrm{~K} / \mathrm{m})$ exists across the material. That is, it is the heat flow per unit area per unit time when the temperature decreases by one degree in unit distance. Thus,

$$
\mathrm{Q}=-\mathrm{kA} \frac{\Delta \mathrm{T}}{\Delta \mathrm{x}}
$$

or

$$
\mathrm{Q}=-\mathrm{kA} \frac{\mathrm{T}_{\mathrm{H}}-\mathrm{T}_{\mathrm{L}}}{1}
$$

where $Q=$ heat flux, $k=$ thermal conductivity, $A=$ crosssectional area, $T_{H}=$ temperature at hot end, $T_{L}=$ temperature at cold end, and $l=$ thickness of material respectively, and the negative sign indicates that the heat flow is positive in the direction of temperature fall.

(ii) Training the Ann Model Using Back Propagation Algorithm

Neural networks have been trained to perform complex functions in various fields including pattern recognition, identification, classification, speech, vision, and control systems. Today, neural networks can be trained to solve problems that are difficult for conventional computers or human beings. Commonly, neural networks are adjusted (or trained), so that a particular input leads to a specific target output. The schematic of such a situation is shown in Fig. 3 where the network is adjusted based on a comparison of the output and the target until the output matches the target. 
Typically, many such input/target pairs are needed to train a neural network.

The back-propagation algorithm was utilized in model training. A hyperbolic-tangent transfer function was also used in all cases. Properly trained back-propagation networks (Fig. 4) tend to give reasonable answers when presented with unfamiliar inputs that have never been seen earlier. Typically, a new input leads to an output similar to the correct output for input vectors used in training that are similar to those being presented. This generalization properly makes it possible to train a network on a representative set of input/target pairs and get good results without training the network on all possible input/output pairs.

The back-propagation algorithm uses the supervised training technique where the network weights and biases are initialized randomly at the beginning of the training phase. For a given set of inputs to the network, the response to each neuron in the output layer is calculated and compared with the corresponding desired output response. The errors associated with desired output response are adjusted in the way that reduces these errors in each neuron from the output to the input layer.

In order to avoid the potential problem of over-training or memorization while employing the back-propagation algorithm, the option of saving the best result is adopted during the selected number of training cycles of 2,000.

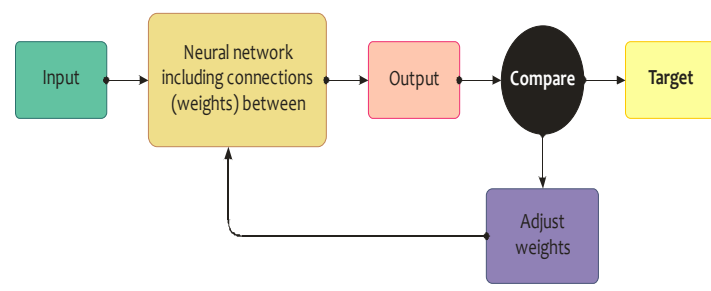

Fig. 3. Adjusting (or training) of a Neural network

(iii) Optimal Configuration of Ann Model for Bakery Products

Upon using the mean relative error (MRE), mean absolute error (MAE) and standard error (SE) as standard criteria, the performances of the various ANN configurations were compared. The mathematical representation of these error parameters are defined in equations 8 to 10 as follow $[10,14]$ :

$$
\begin{aligned}
\mathrm{MAE} & =\frac{1}{\mathrm{n}}\left[\sum_{i=1}^{n}\left(K_{D}-K_{P}\right)\right] \\
\mathrm{MRE}=\frac{1}{\mathrm{n}}\left[\sum_{i=1}^{n}\left(K_{D}-K_{P}\right) / K_{D}\right] & \\
\mathrm{SE} & =\left[\frac{\sqrt{\sum_{i=1}^{n}\left(K_{D}-K_{P}\right)^{2}}}{\mathrm{n}-1}\right]
\end{aligned}
$$

where $n$ is the number of data points, $K_{D}$ and $K_{P}$ are the desired and predicted values of thermal conductivity respectively.

The optimum configuration of the network was chosen by selecting the lower value from the different configuration of the network. It was evidently based upon minimizing the difference between the neural network predicted values and the desired outputs. The datasets of 52 cases obtained from other literature [1] were divided into two sets. The first set consisted of $36(\sim 70 \%)$ cases for training/testing and $16(\sim 30 \%)$ cases for validation (simulation), chosen randomly from the set of 52 cases.

\section{Simulation and Results}

\subsection{Simulation}

Computer simulation of ANN was employed for the purpose of this work using the MATLAB version 7.0.4.365 (R14) Service Pack 2 commercial software package with embedded neural network add-in toolbox.

Several ANN models were simulated (or trained) using the thermo-physical properties datasets of Table 2. The feed-forward network structure with input, output and hidden layers were also used and the generalized network structures are as shown in Fig. 5 and Fig. 6 respectively.

Table 2. Thermal conductivity of dough and bakery products

\begin{tabular}{llllll}
\hline Product & Temp. $\left({ }^{(\mathbf{C})}\right.$ & Moisture content $(\%)$ & $\begin{array}{l}\text { Apparent } \\
\text { Density }\left(\mathbf{k g} / \mathbf{m}^{\mathbf{3}}\right)\end{array}$ & $\begin{array}{l}\text { Thermal Conductivity } \\
(\mathbf{W} / \mathbf{m K})\end{array}$ & Technique \\
\hline Bread & 22 & $28-36$ & $190-210$ & $0.091-0.104$ & Line heat source [19] \\
Bread dough & $-43.5-21$ & $43.5-46.1$ & 1100 & $0.500-0.920$ & Transient hot strip [27] \\
French bread & 22 & 42.0 & 161.4 & 0.0989 & Linear heat source [17] \\
Yellow cake batter & 22 & $35.5-41.5$ & $300-694$ & $0.121-0.223$ & Linear heat source [17] \\
Cup cake batter & $20-104$ & $25-37$ & $272-815$ & $0.068-0.290$ & Linear heat source [1, 2] \\
Tortilla chip & 25 & $1.4-35.6$ & $520-880$ & $0.09-0.23$ & Linear heat source [4] \\
Whole wheat dough & $35-62.5$ & $42.4-46.0$ & $1035-1093$ & $0.221-0341$ & Modified guarded hot plate [15] \\
Baked chapatti & $35-62.5$ & $38-48.7$ & $1050-1200$ & $0.142-0343$ & \\
& & & & & \\
\hline
\end{tabular}




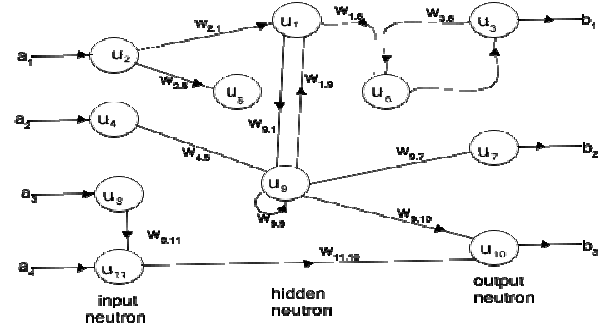

Fig. 4. A back propagation network

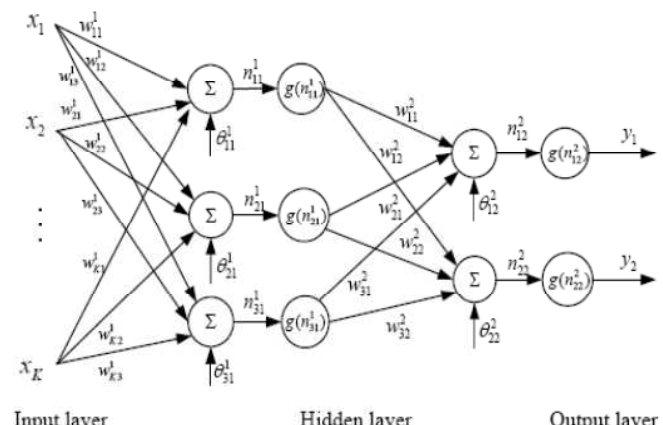

Fig. 5. Generalized multilayer neural network

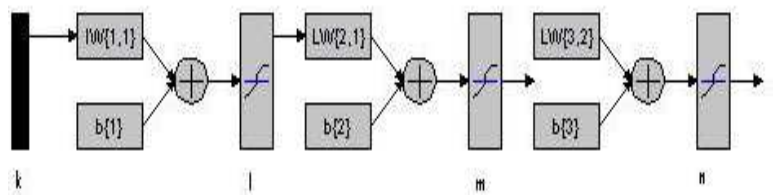

Fig. 6. Generalized multilayer neural network obtained with MATLAB

For the purpose of this work, the input layer consisted of 3 neurons which corresponded to a product's moisture content, temperature and apparent density respectively, while the output layer had 1 neuron representing the thermal conductivity. The number of hidden layers and neurons within each hidden layer can be varied based on the complexity of the problem and dataset. Moreover, the number of hidden layers was varied from 1 to 2 . The neurons within each of these layers were varied from 2 to 16 with increments of 2 . This resulted in a total of 16 networks.

\subsection{Results of Simulation}

Once a given ANN configuration was trained using the input data, its performance was evaluated using the same dataset. The analysis was repeated several times. The ANN configuration (out of 16) that minimized the three error measures: MRE, MAR and SE, was selected as the optimum.

The error measures associated with different ANN configurations for prediction of thermal conductivity are presented in Table 3. The optimal ANN configuration included 2-hidden layers with 8 neurons in each layer. The MAE, MRE and SE for this optimal configuration were $0.0054 \mathrm{~W} / \mathrm{mK}, 4.8776 \times 10^{-4} \mathrm{~W} / \mathrm{mK}\left(4.8776 \times 10^{-2} \%\right)$ and $0.0015 \mathrm{~W} / \mathrm{mK}$ respectively (shown highlighted below).
Table 3. Error parameters in the prediction of thermal conductivity with different neural network configurations

\begin{tabular}{lllll}
\hline $\begin{array}{l}\text { No. of } \\
\text { hidden } \\
\text { layers }\end{array}$ & $\begin{array}{l}\text { No. of } \\
\text { neurons in } \\
\text { each hidden } \\
\text { layer }\end{array}$ & MRE $(\%)$ & $\begin{array}{l}\text { MAE } \\
(\mathbf{W} / \mathbf{m K})\end{array}$ & $\begin{array}{l}\text { SE } \\
(\mathbf{W} / \mathbf{m K})\end{array}$ \\
\hline 1 & 2 & 0.17 & 0.0199 & 0.0046 \\
1 & 4 & 4.2 & 0.2212 & 0.0970 \\
1 & 6 & 0.035077 & 0.0038 & 0.0011 \\
1 & 8 & 0.18 & 0.0191 & 0.0050 \\
1 & 10 & 0.03388 & 0.0034 & 0.0011 \\
1 & 12 & 0.26 & 0.0297 & 0.0073 \\
1 & 14 & 0.090897 & 0.0149 & 0.0041 \\
1 & 16 & 0.12 & 0.0115 & 0.0038 \\
2 & 2 & 0.24 & 0.0298 & 0.0075 \\
2 & 4 & 0.41 & 0.0641 & 0.0125 \\
2 & 6 & 0.37 & 0.0606 & 0.0121 \\
2 & 8 & 0.048776 & 0.0054 & 0.0015 \\
2 & 10 & 0.29 & 0.0441 & 0.0106 \\
2 & 12 & 0.22 & 0.0382 & 0.0095 \\
2 & 14 & 0.17 & 0.0166 & 0.0047 \\
2 & 16 & 0.22 & 0.0323 & 0.0072 \\
\hline
\end{tabular}

Table 4. Error prediction for 1 hidden layer with their corresponding neurons

\begin{tabular}{llll}
\hline $\begin{array}{l}\text { No. of neurons in } \\
\text { each hidden layers }\end{array}$ & $\begin{array}{l}\text { MRE }(\% \\
\left.\mathbf{x 1 0}^{-\mathbf{2}}\right)\end{array}$ & $\begin{array}{l}\text { MAE }(\mathrm{W} / \mathrm{mK} \\
\left.\mathbf{x 1 0}^{-\mathbf{3}}\right)\end{array}$ & $\begin{array}{l}\mathrm{SE}(\mathrm{W} / \mathbf{m K} \\
\left.\mathbf{x 1 0 ^ { - 4 }}\right)\end{array}$ \\
\hline 2 & 17 & 19.9 & 46 \\
4 & 420 & 221.2 & 970 \\
6 & 3.5 & 3.8 & 11 \\
8 & 18 & 19.1 & 50 \\
10 & 3.4 & 3.4 & 11 \\
12 & 26 & 29.7 & 73 \\
14 & 9.1 & 14.9 & 41 \\
16 & 12 & 11.5 & 38 \\
\hline
\end{tabular}

Table 5. Error prediction for 2 hidden layers with their corresponding neurons

\begin{tabular}{llll}
\hline $\begin{array}{l}\text { No. of neurons in } \\
\text { each hidden layers }\end{array}$ & $\begin{array}{l}\text { MRE }(\% \\
\left.\mathbf{x 1 0 ^ { - 2 }}\right)\end{array}$ & $\begin{array}{l}\text { MAE }(W / m K \\
\left.\mathbf{x 1 0}^{-3}\right)\end{array}$ & $\begin{array}{l}\text { SE }(W / m K \\
\left.\mathbf{x 1 0}^{-4}\right)\end{array}$ \\
\hline 2 & 24 & 29.80 & 75 \\
4 & 41 & 64.10 & 125 \\
6 & 37 & 60.60 & 121 \\
8 & 4.88 & 5.40 & 15 \\
10 & 29 & 44.10 & 106 \\
12 & 22 & 38.20 & 95 \\
14 & 17 & 16.60 & 47 \\
16 & 22 & 32.30 & 72 \\
\hline
\end{tabular}

\subsection{Discussion of Results}

Tables 3 and 4 show the error parameters in the prediction of thermal conductivity for 1- and 2-hidden layers with 2 to 16 neurons in each respective hidden layer. The corresponding charts of Tables 4 and 5 are shown in Figs. 7and 8 respectively. For each of the corresponding diagrams, there was a considerable and first-rate agreement 
between the predicted and desired/targeted values of thermal conductivities for different parameters of MAE, MRE and SE.

It can be concluded that the predicted thermal conductivity is good, efficient and credible prediction for thermal conductivity of bakery products.

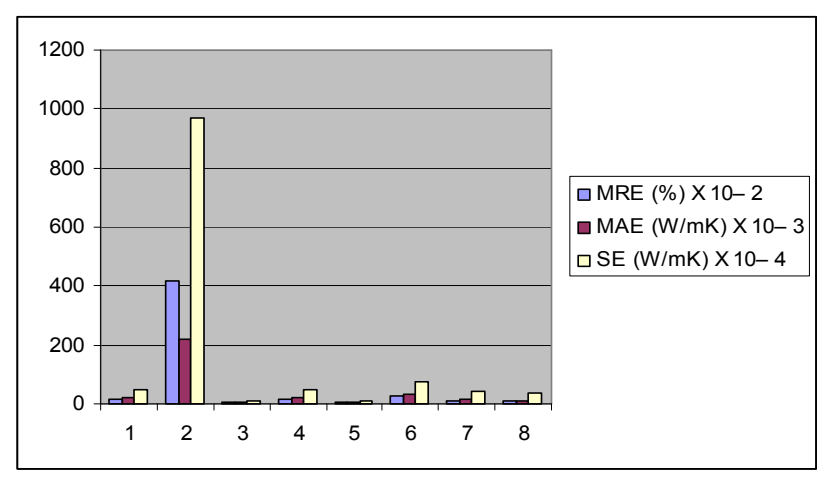

Fig. 7. Corresponding chart showing the error values for 1-hidden layer

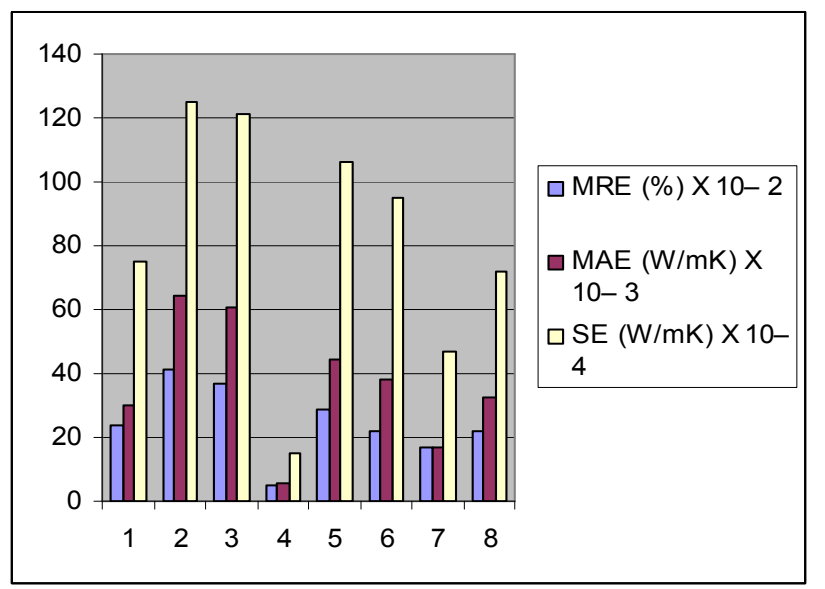

Fig. 8. Corresponding chart showing the error values for 2-hidden layers

\subsection{Training, Validation and Prediction}

\subsubsection{Training Dataset}

To reveal the credibility of prediction (with the training data set) from the optimal ANN, predicted values of thermal conductivity are plotted against the desired/targeted values of thermal conductivity as shown in Fig. 9. Similarly, that of the simplest ANN model is shown in Fig. 10. These two figures showed straight line curves, demonstrating correlation between the predicted and targeted thermal conductivities when the points are joined together (best fitpoints). The results demonstrate a very good agreement between the predicted and the desired values of thermal conductivity. Considering the inherent variation in the input dataset, the simplest ANN configuration can be considered. The simplest ANN model with 1-hidden layer and 10 neurons predicted thermal conductivity with a $0.34 \%$ (0.0034 W/mK) MAE, $3.386 \times 10^{-4} \mathrm{~W} / \mathrm{mK}$ MRE and $0.0011 \mathrm{~W} / \mathrm{mK}$ SE being the least of all values of the error measures.

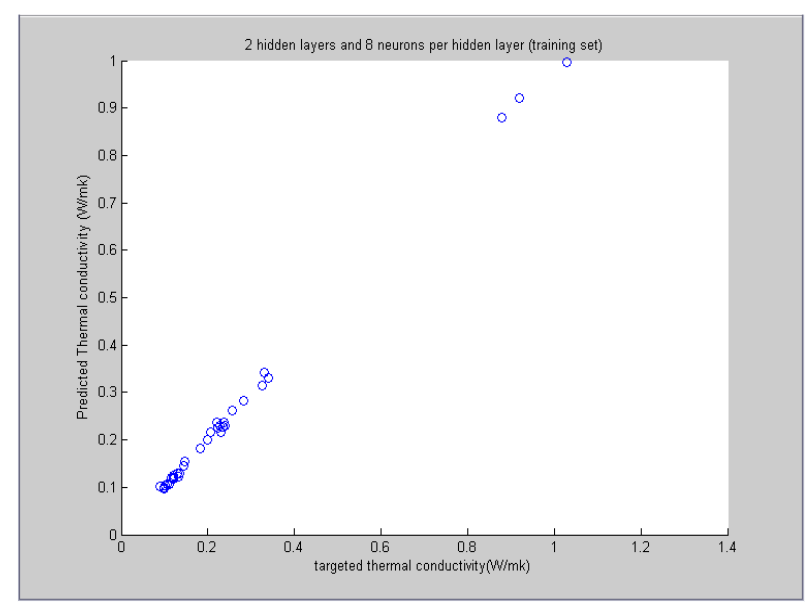

Fig. 9. 2-hidden layers with 8 neurons

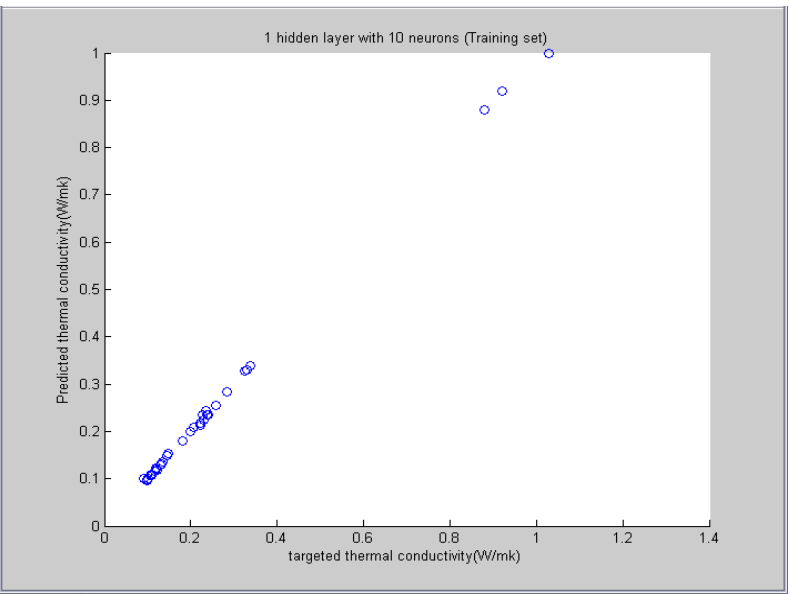

Fig. 10. 1-hidden layers with 10 neurons

\subsubsection{Validating Dataset}

The performance of the optimal neural network was validated using a second dataset consisting of 16 cases not previously used in the training. Thermal conductivity values with an MRE of $0.0217 \mathrm{~W} / \mathrm{mK}$, MAE of 0.1544 $\mathrm{W} / \mathrm{mK}$ and SE of $0.0156 \mathrm{~W} / \mathrm{mK}$ for 2-hidden layers with 8 neurons were predicted. The graph between the predicted and the desired values of thermal conductivity for this model is shown in Fig. 11. There seemed to be no correlation between the predicted and the targeted thermal conductivities because of the few number of cases (16) used for the validating dataset, as compared to that used for the training set (36).

This data set was also used with the simplest ANN configuration of one hidden layer consisting of ten neurons. The model predicted thermal conductivity with a $4.73 \%$ (or $0.0473 \mathrm{~W} / \mathrm{mK}$ ) MRE, $25.6 \%$ (or $0.2559 \mathrm{~W} / \mathrm{mK}$ ) MAE and $11.6 \%$ (or $0.1163 \mathrm{~W} / \mathrm{mK}$ ) SE. The graph between the predicted and the targeted values of thermal conductivity for the simplest ANN model is shown in Fig. 12. Similar reasons could also be given for the non-correlation between the predicted and targeted thermal conductivities for the validating dataset, that is, fewer numbers of cases (16) were used. 


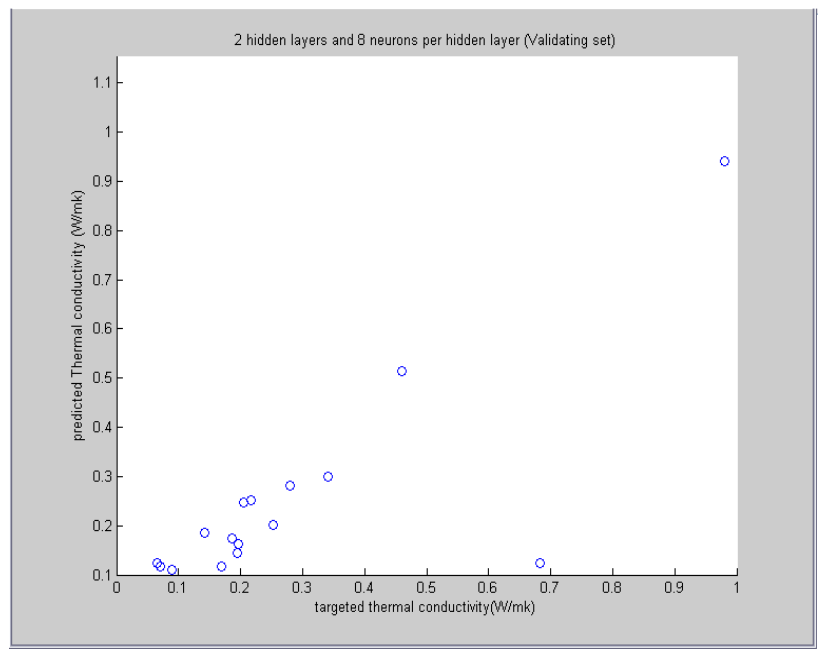

Fig. 11. 2-hidden layers with 8 neurons

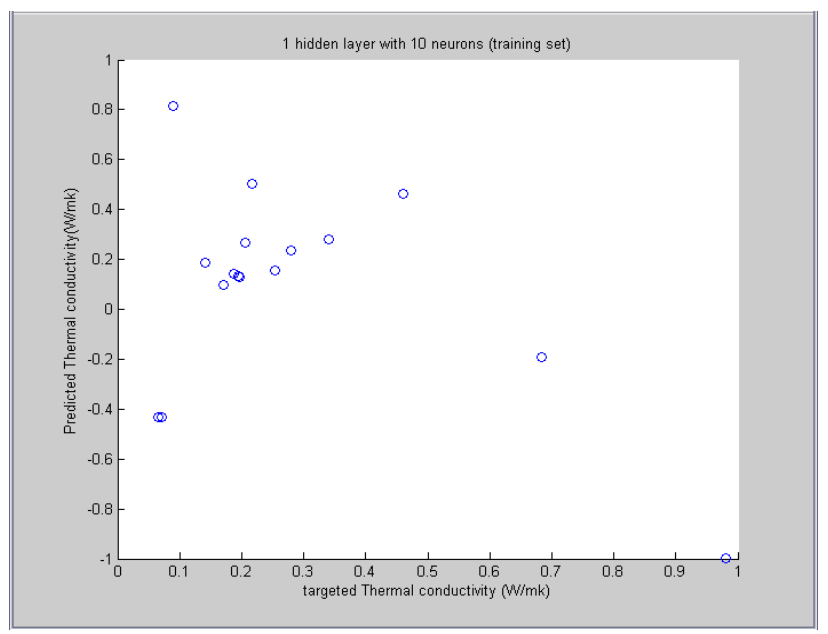

Fig. 12. 1-hidden layer with 10 neurons

\subsubsection{Predicted and Targeted Thermal Conductivities}

The thermal conductivity of the bakery products was modelled by simulation. As a result, both the predicted and targeted/desired thermal conductivity values are plotted separately against each of the three dependent variables as a function of moisture content $(\%)$, temperature $\left({ }^{\circ} \mathrm{C}\right)$ and apparent density $\left(\mathrm{kg} / \mathrm{m}^{3}\right)$. The corresponding curves were obtained for the optimal ANN configuration; 2-hidden layers with 8 neurons (Figs. 13 to 15) and the simplest ANN configuration; 1-hidden layer with 10 neurons (Figs. 16 to 18$)$.

For each of the corresponding diagrams, black triangles $(\pi)$ represent the predicted thermal conductivity while purple stars (*) represent the targeted/desired thermal conductivity. Figs. 13 to 18 showed a considerable and first-rate agreement between the predicted and desired/targeted values of thermal conductivities as the points considered (shown as triangles and stars) intersected at some locations on the curves and nearly intersected at other locations.

Since most of the triangle-points of the predicted values of thermal conductivity intersected with most of the starpoints of the thermal conductivity, it can be concluded that the predicted thermal conductivity is a good, efficient and credible prediction in comparison to the desired/targeted value (Figs. 13 to 18 ) for both the optimal and simplest ANN models.

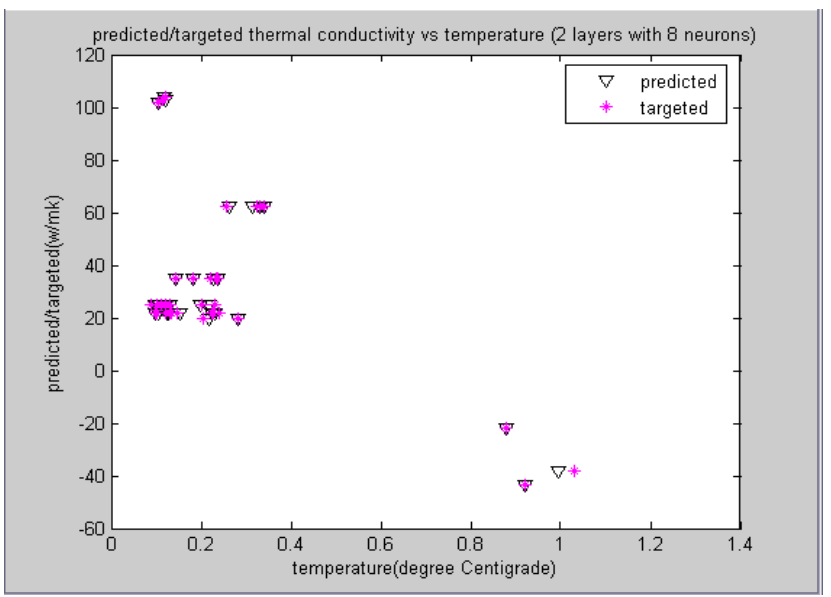

Fig. 13. Thermal conductivities vs. Temperature (2 hidden layers with 8 neurons)

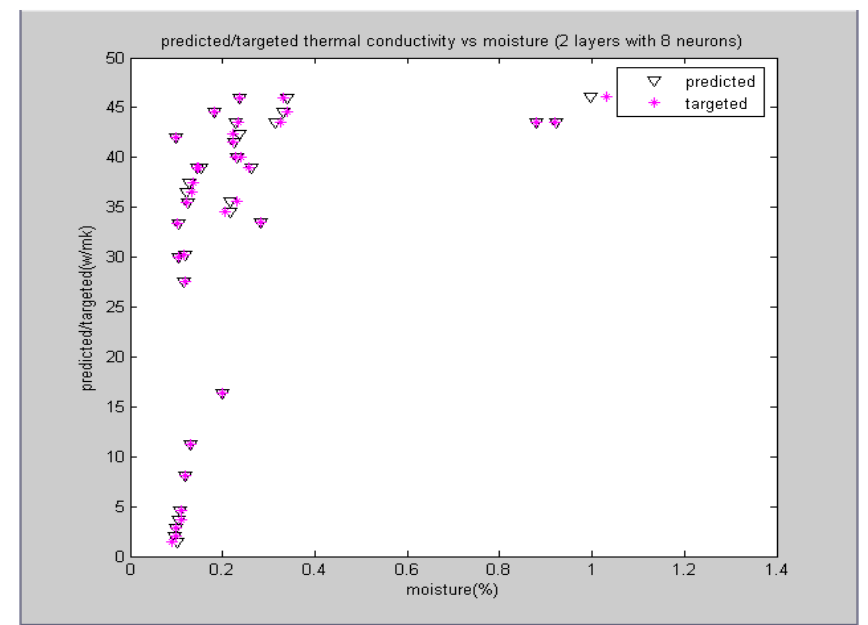

Fig. 14. Thermal conductivities vs. Moisture content (2 hidden layers with 8 neurons)

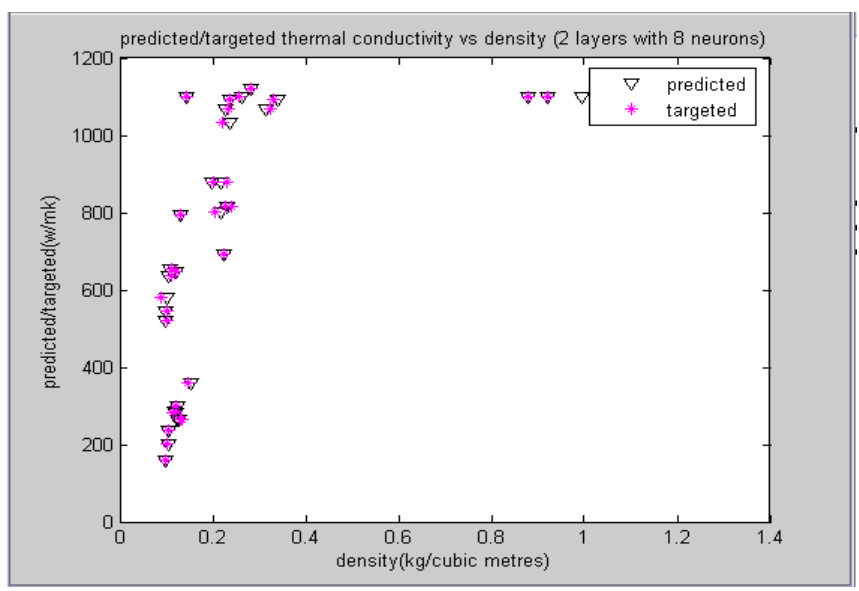

Fig. 15. Thermal conductivities vs. Apparent density (2 hidden layers with 8 neurons) 


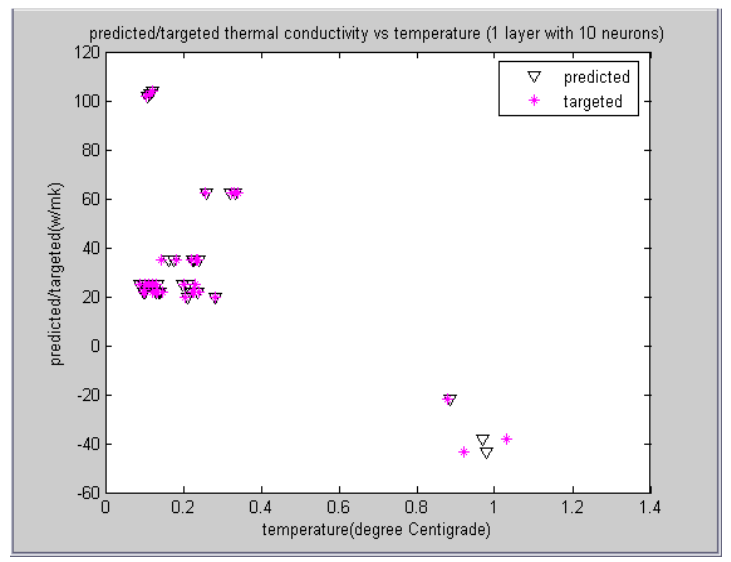

Fig. 16. Thermal conductivities vs. Temperature (1 hidden layer with 10 neurons)

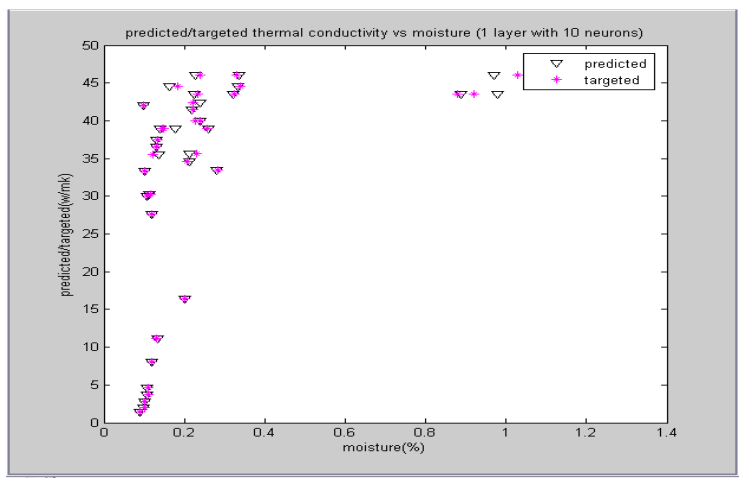

Fig. 17. Thermal conductivities vs. Moisture content (1 hidden layer with 10 neurons)

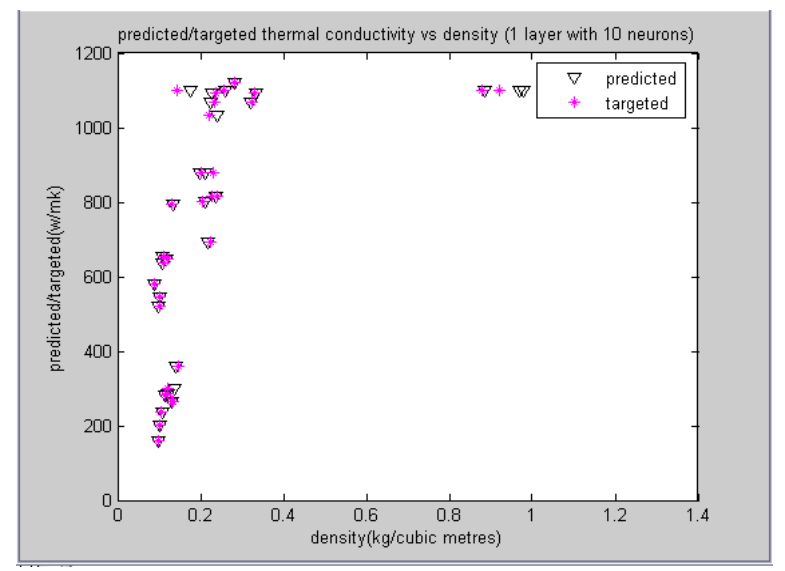

Fig. 18. Thermal conductivities vs. Apparent density (1 hidden layer with 10 neurons)

Finally, there is a great disagreement or deviation between the predicted values of thermal conductivity and the targeted values when plotted against density for an ANN model that has 1 hidden layer with 4 neurons, as shown in Fig. 19. Similarly, when the predicted and targeted values of thermal conductivity are plotted against moisture content for an ANN model that has 2 hidden layers with 12 neurons, there was no agreement, as shown in Fig. 20. These ANN models are said to be inefficient models because of the non-intersection of most of their triangles and stars points.

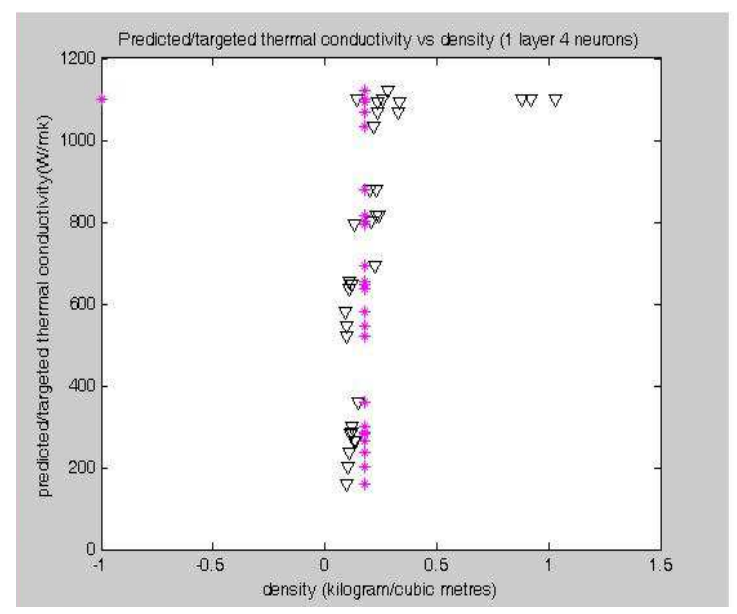

Fig. 19. Thermal conductivities vs. Apparent density (1 hidden layer with 4 neurons)

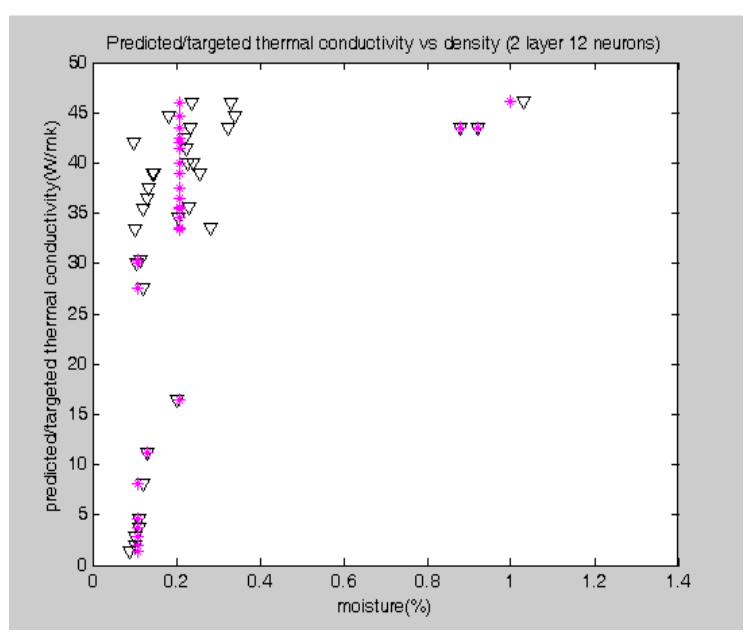

Fig. 20. Thermal conductivities vs. Moisture content (2 hidden layers with 12 neurons)

\section{Conclusion}

In this paper, an ANN model was developed for calculating the thermal conductivity of a variety of bakery products under a wide range of conditions of moisture content, temperature and apparent density. The optimal model consisted of 2-hidden layers with eight neurons in each hidden layer, and was able to produce thermal conductivity values with a MAE of $54 \times 10^{-4} \mathrm{~W} / \mathrm{mK}$, MRE of $4.878 \times 10^{-4} \mathrm{~W} / \mathrm{mK}$ and a SE of $15 \times 10^{-4} \mathrm{~W} / \mathrm{mK}$ (see Table 3). However, the simplest ANN model has 1-hidden layer with 10 neurons. This also showed a good prediction with a MRE of about $3.388 \times 10^{-4} \mathrm{~W} / \mathrm{mK}$, MAE of $34 \mathrm{X}$ $10^{-4} \mathrm{~W} / \mathrm{mK}$ and SE of $11 \times 10^{-4} \mathrm{~W} / \mathrm{mK}$ (see Table 4).

From these values, it can be deduced and concluded that the simplest ANN model (with 1-hidden layer and 10 neurons), when compared with the optimal ANN model (with 2-hidden layers and 8 neurons in each hidden layer) has smaller mean relative error,smaller mean absolute error and lesser standard error. Therefore, this model performs better accordingly. 


\section{References}

[1] Baik, O. D., Marcotte, M., Sablani, S. S., and Castaigne, F. (2001): "Thermal and physical properties of Bakery products"; Critical Review in Food Science and Nutrition. CRC Press LLC; 2001.

[2] Baik, O. D., Marcotte, M., Sablani, S. S., and Castaigne, F. (2006): "Modelling the Thermal properties of a Cup cake during Baking"; Journal of Food Science Volume 64, Issue 2, Pgs. 295 - 299; 2006.

[3] Ajasa, A. A., Akinyemi, L. A., Shoewu, O. O. and Adenowo, A. A. (2014): "Exploitation of Artificial Neural Networks to predict the Thermal Conductivity of Food Products in Nigeria"; American Journal of Engineering Research (AJER); Volume 03, Issue 03, Pgs 22 - 29.

[4] Shyam Sablani, S and Shafiur Rahman, M. (2003), "Using neural networks to predict thermal conductivity of food as a function of moisture content, temperature and apparent porosity"; Food Research International. Elsevier. Volume 36, Issue 6, 2003, Pgs. 617 - 623; 2003.

[5] Rask, C. (1989): "Thermal properties of dough and bakery products: A review of published data"; Journal of Food Engineering, 9, Pgs 167 - 193; 1989

[6] Hussain, A. M. and Rahman, M. S. (1999): “Thermal conductivity prediction of fruits and vegetables using neural networks"; International Journal of Food Properties; 2(2); 1999.

[7] Ni, H., and Gunasekaran, S. (1998): "Food quality prediction with neural networks"; Food Technology, 52(10), Pgs $60-65 ; 1998$.

[8] Sablani, S. S., Marcotte, M., Baik, O. D., and Castaigne, F. (1998): "Modelling of simultaneous heat and water transport in the baking process"; Lebensmittel Wissenchaft and Technologie, 31, Germany; Pgs 201 - 209; 1998.

[9] Linko, P., and Zhu. Y. H. (1991): "Neural network programming in bioprocess variable estimation and state prediction"; Journal of Biotechnology, 21(3), Pgs 253-270; 1991.

[10] Hornik, K., Stinchombe, M. and White, H.: (1989): "Multilayer feed-forward network and universal approximator. Neural Network", 2, Pgs. 359 - 366; 1989.

[11] Myhara, R. M., Sablani, S. S., Al-Alawi, S. M., and Taylor, M. S. (1998):"Water sorption isotherms of dates: Modelling using GAB equation and artificial neural network approaches"; Lebensmittel Wissenschaft und Technologies, 31(7/8), Germany; Pgs 699 - 706; 1998.

[12] Murakami, E. G., and Okos, M. R. (1989): “Measurement and Prediction of thermal properties of foods". In R. P. Singh, and A. G. Medina (Eds.), Food Properties Computeraided Engineering of Food Processing System; Pgs. 3 - 48; Norwell, MA; Kluwer Academic Publishers, 1989.

[13] Xie, G. and Xiong, R. (1999): "Use of hyperbolic and neural network models in modelling quality changes of dry peas in long time cooking"; Journal of Food Engineering, 41, (3/4), Pgs. $151-162 ; 1999$.

[14] Bishop, M. C. (1994): "Neural networks and their applications"; Review in Scientific Instruments, 64(6), Pgs. $1803-1831 ; 1994$

[15] Ruan, R., Almaer, S., \& Zhang, J. (1995): "Prediction of dough rheological properties using neural networks"; Cereal Chemistry, 72(3), Pgs $308-311 ; 1995$.

[16] Rogers, G. F. C and Mayhew, Y. R. (1989): "Engineering Thermodynamics”; ELBS 3/e, Pgs. 469 - 502; 1989.

[17] Sweat, V. E. (1985): "Thermal properties of low and intermediate moisture food"; ASHRAE Transaction, 91, Pgs. $369-389 ; 1985$.

[18] Fang, Q., Bilby, G., Haque, E., Hanna, M. A., and Spillman, C. K. (1998):" Neural network modelling of physical properties of ground wheat"; Cereal Chemistry, 75(2),Pgs $251-253 ; 1998$.

[19] Bakshi, S. A. and Yoon, J. (1984): "Thermophysical properties of bread rolls during baking"; Lebensmittel Wissenschaft und Technologie, 17, Pgs. 90 - 93; 1984.

[20] Sablani, S. S. and Shayya, W. H. (2001): “Computerization of Stumbo's method of thermal process calculations using neural networks"; Journal of Food Engineering, 47, Pgs. $233-240 ; 2001$.

[21] Christina Rask (1989): "Thermal properties of dough and bakery products"; A review of published data. Journal of Food Engineering. Volume 9, Issue 3, 1989, Pgs. 167 - 193; 1989.

[22] Kulacki, F. A. and Kennedy, S. C. (1978): "Measurement of the thermo-physical properties of common cookie dough"; Journal of Food Science; 43, Pgs. 380 - 384; 1978.

[23] Buhri, A. B. and Singh, R. P. (1993): "Measurement of food thermal conductivity using differential scanning calorimetry"; Journal of Food Science; 58, Pgs. 1145 - 1147, 1993.

[24] Zhou, L., Puri, V. M. and Anantheswaran, R. C. (1994): "Measurement of coefficients for simultaneous heat and mass transfer in food products"; Drying Technology; 12; Pgs. 607 - 627; 1994.

[25] Vos, B. H. (2005):"Measurements of thermal conductivity by a non-steady state method"; Applied Science Research; A5; Pgs. 425 - 438; 2005.

[26] Gustafsson, S. E., Karawacki, E. and Khan, M. N. (2001): "Transient hot-strip method for simultaneously measuring thermal conductivity and thermal diffusivity of solids and fluids"; Journal of Applied Physics; 12; Pgs. 1411 - 1421; 2001.

[27] Lind, I. (1998): "Thawing of minced meat and dough: thermal data and mathematical modeling"; Pro. Int. Symp. On progress in food preservation process; Vol. 1, CERIA, Brussels, Belgium; Pgs. 101 - 111; 1998. 